Military Technical College

Kobry El-Kobba

Cairo, Egypt

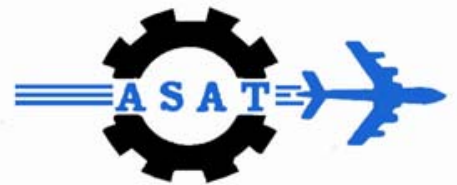

12-th International Conference

on

Aerospace Sciences \&

Aviation Technology

\title{
SIMULTANEOUS TRAJECTORY MANIPULATION OF A DUAL-ARM ROBOT USING SLIDING MODE CONTROL
}

\author{
Nurkan Yagiz ${ }^{\star}$ Yuksel Hacioglu* and Yunus Ziya Arslan ${ }^{\star}$
}

\begin{abstract}
Dual-arm robotic system for load transportation in hazardous environments is considered in this study. First, physical model of the robot arms with the load is presented and related equations of motion are obtained. Friction forces arising on the contact points between the load and robot arm tips are also taken into account. Then, robust non-chattering sliding mode control is used in order to obtain the joint torques, which makes the robot track the trajectory and move the load to its new location. Finally, numerical results are presented. These results verify that this type of robot systems with proposed sliding mode control method can be used in hazardous applications safely.
\end{abstract}

\section{KEY WORDS}

Sliding mode control, Dual arm robot, Trajectory Manipulation, Load transportation, Hazardous Environments

\footnotetext{
* Department of Mechanical Engineering, Faculty of Engineering, Istanbul University, 34320 Avcilar, Istanbul, Turkey
} 


\section{INTRODUCTION}

In industrial automation systems, to handle large objects and assemble complex industrial parts with higher precision and confidence, dual arm cooperative robot systems are widely used. [1]. Furthermore, during the hazardous tasks, such as transportation of the active uranium in nuclear power plants or performing disposal of explosive ordnances, dual-arm robot systems are frequently preferred [2]. Using dual arm robot is more advantageous than single arm robot due to the requirement of less torque which is to be applied on the joint for accomplishing the same task. However, the main disadvantage of using two-arm robot is arising from the necessity of more complex mechanic analysis and control strategy design [3].

It is necessary to employ a controller to have the coordinated arms perform synchronously while tracking their given trajectories with high performance. Uchiyama et al. [4] applied a hybrid control technique to a two arm industrial robot for realizing human skill by using artificially built mechanical arms. Laroussi et al. [5] considered using linear state feedback for stabilization and control of the two planar robots in lifting a load and transporting it to a new location. However, unexpected disturbances and obstacles can occur in the workspace or task environment of the robot system while performing. In these circumstances, in order to maintain the desired motion trajectory, it is necessary to use a robust and reliable controller. Thus sliding mode controller is preferred in this study.

After a paper by Utkin [6] sliding mode control has become widespread within control theory practitioners and academicians. In this control method, states of the system are forced to reach a predefined sliding surface and then they are kept on this surface and a sliding motion takes place. During the sliding motion the system is insensitive to parameter variations and external disturbances. Because of its robust behavior, this control method has found a wide range of application areas in industry like, robotic manipulator control [7], control of mobile robots [8], process control [9], and flight control [10].

\section{PHYSICAL MODEL}

The dual arm robotic system consisting of two planar robot arms with actuating motors at revolute joints is seen in Figure 1. The system has four degrees of freedom (DoF). When the robots handle the object, because of the constraints, the DoF of the system reduces to two. Note that the load is not allowed to rotate. In physical model of the robot system, $m_{i}, I_{i}$ and $L_{i}$ represents the mass, mass moment of inertia and length of the related links. $k_{i}$ is the distance of the mass center and $\theta_{i}$ is the joint angle of the related links. Also, there are viscous frictions on all of the joints denoted by $b_{i}$. Numerical values of the parameters are given in Appendix. 
The robot arms move in the horizontal $x y$-plane and gravity acts in negative $z$ direction. There are two periods in the motion of the robots. First, robots start from their home position and move towards the rectangular load. Then, in the second part robots handle the load and move it to its new position while tracking the given trajectory.

In order to perform transportation task, robot applies forces $F_{1}, F_{2}$ from arm tips to the load (Figure 2). Friction-assisted handling prevents slipping of the load from the contact points during motion. Friction forces $F_{s 1}, F_{s 2}$ and their components $F_{s 1 y}, F_{s 1 z}, F_{s 2 y}, F_{s 2 z}$ between arm tips and load surface are shown in Figure 2. Here $\mu$ represents the coefficient of dry friction. In this study it is aimed that the load is moved without rotation. Thus, $F_{s 1 y}$ and $F_{s 2 y}$ are equal preventing the rotation about $z$-axis. Similarly, $F_{s 1 z}$ and $F_{s 2 z}$ are equal since there is no rotation about $y$-axis, too. Then:

$$
\begin{aligned}
& F_{s 1 y}=F_{s 2 y} \\
& F_{s 1 z}=F_{s 2 z}=m g / 2
\end{aligned}
$$

Equations of motion after handling the load are given below,

$$
\begin{aligned}
& \ddot{\theta}_{1}\left(A_{1}+A_{2}+2 A_{3} \cos \theta_{2}\right)+\ddot{\theta}_{2}\left(A_{2}+A_{3} \cos \theta_{2}\right)-A_{3} \sin \theta_{2}\left(\dot{\theta}_{2}^{2}+2 \dot{\theta}_{1} \dot{\theta}_{2}\right)+b_{1} \dot{\theta}_{1} \\
= & u_{1}-F_{1}\left[L_{1} \sin \theta_{1}+L_{2} \sin \left(\theta_{1}+\theta_{2}\right)\right]-F_{s 1 y}\left[L_{1} \cos \theta_{1}+L_{2} \cos \left(\theta_{1}+\theta_{2}\right)\right] \\
& A_{2} \ddot{\theta}_{2}+\ddot{\theta}_{1}\left(A_{2}+A_{3} \cos \theta_{2}\right)+A_{3} \dot{\theta}_{1}^{2} \sin \theta_{2}+b_{2} \dot{\theta}_{2}=u_{2}-F_{1} L_{2} \sin \left(\theta_{1}+\theta_{2}\right) \\
- & F_{s 1 y} L_{2} \cos \left(\theta_{1}+\theta_{2}\right) \\
& \ddot{\theta}_{3}\left(A_{4}+A_{5}+2 A_{6} \cos \theta_{4}\right)+\ddot{\theta}_{4}\left(A_{5}+A_{6} \cos \theta_{4}\right)-A_{6} \sin \theta_{4}\left(\dot{\theta}_{4}^{2}+2 \dot{\theta}_{3} \dot{\theta}_{4}\right)+b_{3} \dot{\theta}_{3} \\
= & u_{3}+F_{2}\left[L_{3} \sin \theta_{3}+L_{4} \sin \left(\theta_{3}+\theta_{4}\right)\right]-F_{s 2 y}\left[L_{3} \cos \theta_{3}+L_{4} \cos \left(\theta_{3}+\theta_{4}\right)\right] \\
& A_{5} \ddot{\theta}_{4}+\ddot{\theta}_{3}\left(A_{5}+A_{6} \cos \theta_{4}\right)+A_{6} \dot{\theta}_{3}^{2} \sin \theta_{4}+b_{4} \dot{\theta}_{4}=u_{4}+F_{2} L_{4} \sin \left(\theta_{3}+\theta_{4}\right) \\
- & F_{s 2 y} L_{4} \cos \left(\theta_{3}+\theta_{4}\right)
\end{aligned}
$$

where $A_{i}$ are constant coefficients given in Appendix.

Since the $x, y$ coordinates of the center of mass are common in defining the trajectory of both arms, the DoF of the overall system reduces to two. The dynamic equations of the load are:

$$
m \ddot{x}_{m}=F_{2}-F_{1}
$$




$$
m \ddot{y}_{m}=2 F_{s 1 y}=2 F_{s 2 y}
$$

and the expressions for friction forces are:

$$
\begin{aligned}
& {F_{s 1 y}}^{2}+\left(\frac{m g}{2}\right)^{2}<\left(\mu F_{1}\right)^{2} \\
& F_{s 2 y}{ }^{2}+\left(\frac{m g}{2}\right)^{2}<\left(\mu F_{2}\right)^{2}
\end{aligned}
$$

\section{CONTROLLER DESIGN}

In sliding mode controlled systems, the control input is changed intentionally according to predefined rules, which drives the system states towards a sliding surface and constrain to stay over this surface. Therefore, there are two parts in the design stage of this controller. The first one is definition of the sliding surface in the state space, and the second one is obtaining the control law in order to construct and maintain such a sliding motion. During the sliding motion the system is insensitive to parameter variations and external disturbances.

The state space form of a non-linear dynamic system can be written as

$$
\dot{\boldsymbol{x}}=\boldsymbol{f}(x)+[B] \boldsymbol{u}
$$

where $\boldsymbol{x}=\left[x_{1}, \ldots, x_{n}, x_{n+1}, \ldots, x_{2 n}\right]^{T}$. The second half of the states is the time derivatives of the first half for mechanical systems, respectively. $2 n$ is the number of the states. In equation (11), $f(x)$ is the $2 n \times 1$ vector of the state equations without the control inputs, $\boldsymbol{u}$ is $n \times 1$ generalized torque input vector and $[B]$ is $2 n \times n$ matrix that its elements are the coefficients of the generalized control inputs in the state equations. The sliding surface is defined as follows:

$$
S=\{\boldsymbol{x}: \boldsymbol{\sigma}(x, t)=0\}
$$

For a control system, the sliding surface can be selected as

$$
\sigma=[G] \Delta x
$$

Here

$$
\Delta \boldsymbol{x}=\boldsymbol{x}_{r}-\boldsymbol{x}=\left[\begin{array}{ll}
\boldsymbol{e} & d \boldsymbol{e} / d t
\end{array}\right]^{T}
$$


is the difference between the reference value and system response. $[G]$ includes the sliding surface slopes. For overall stability, the following Lyapunov function candidate has to be positive definite and its derivative has to be negative semi-definite.

$$
\begin{aligned}
& \boldsymbol{v}(\boldsymbol{\sigma})=\frac{\boldsymbol{\sigma}^{T} \boldsymbol{\sigma}}{2}>0 \\
& \frac{d \boldsymbol{v}(\boldsymbol{\sigma})}{d t}=\frac{\dot{\boldsymbol{\sigma}}^{T} \boldsymbol{\sigma}}{2}+\frac{\boldsymbol{\sigma}^{T} \dot{\boldsymbol{\sigma}}}{2} \leq 0
\end{aligned}
$$

If the limit condition is applied to equation (16), then

$$
\frac{d \boldsymbol{\sigma}}{d t}=\frac{d \boldsymbol{\phi}(t)}{d t}-[G] \frac{d \boldsymbol{x}}{d t}=0
$$

where

$$
\phi(t)=[G] x_{r}
$$

From equation (11) and equation (13)

$$
\frac{d \boldsymbol{\phi}(t)}{d t}-[G]\left(\boldsymbol{f}(x)+[B] \boldsymbol{u}_{e q}\right)=0
$$

$\boldsymbol{u}_{e q}$ is the equivalent control torque input vector for the limit case. Finally equivalent control is found as below,

$$
\boldsymbol{u}_{e q}(t)=[G B]^{-1}\left(\frac{d \boldsymbol{\phi}(t)}{d t}-[G] \boldsymbol{f}(x)\right)
$$

Equivalent control is valid only on sliding surface. Thus, an additional term should be defined to pull the system to the surface. Since the design with classical Lyapunov derivative suffers from chattering, a new derivative candidate is proposed in this study to have a chattering free control. For this purpose, the derivative of the Lyapunov function can be selected as follows.

$$
\dot{v}=-\sigma^{T}[\Gamma] \boldsymbol{\sigma}<0
$$

By equating (18) to (23) and carrying out necessary manipulations, total control input is found as

$$
\boldsymbol{u}(t)=\boldsymbol{u}_{e q}(t)+[G B]^{-1}[\Gamma] \boldsymbol{\sigma}
$$


$[G B]^{-1}$ is always invertible and equal to mass matrix for mechanical systems. [ $[\Gamma]$ is a positive definite matrix, and value of terms are decided by trial at the design stage. However, if the knowledge of $\boldsymbol{f}(x)$ and $[B]$ are not well known, the equivalent calculated control inputs will be completely different from the actual equivalent control inputs. Thus, in this study, it is assumed that the equivalent control is the average of the total control [11].

$$
\hat{\boldsymbol{u}}_{e q}(t)=\frac{1}{\tau s+1} \boldsymbol{u}(t-\delta t)
$$

Finally the non-chattering control input results in,

$$
\boldsymbol{u}(t)=\hat{\boldsymbol{u}}_{e q}(t)+[G B]^{-1}[\Gamma] \boldsymbol{\sigma}
$$

\section{NUMERICAL RESULTS}

Numerical results for two-arm robot system are presented in this section. The parameters of the designed controller are given in Appendix.

There are two stages in the motion of the robot arms namely approaching and transportation stages. Initially the robot arms are at rest and the corresponding initial values of the joints are $\theta_{1}(0)=\pi / 12, \theta_{2}(0)=\pi / 3, \theta_{3}(0)=11 \pi / 12$ and $\theta_{4}(0)=-\pi / 3$. In the first part of the motion, the robots are approaching to the load and in the second part of the motion the robot arms handle the load and transport it to its new location. The reference trajectories for the coordinates of the load center are defined in equation (25) and (26). The initial and final coordinates of the load are given in Appendix. The approaching motion is accomplished until $2^{\text {nd }}$ second and the transportation starts after that moment.

$$
\begin{aligned}
& x_{m r}(t)=x_{f}+\left(x_{i}-x_{f}\right) e^{-50\left(t-t_{t}\right)^{3}} \\
& y_{m r}(t)=y_{f}+\left(y_{i}-y_{f}\right) e^{-50\left(t-t_{t}\right)^{3}}
\end{aligned}
$$

The reference angles for the controllers are obtained by inverse kinematics using the desired trajectory for the load. These reference angles and the actual sliding mode controlled joint angles are given in Figure 3. It is seen from this figure that both of the robot arms track their trajectory successfully since the reference and actual angle values overlap.

The tracking errors for the related joint angles are presented in Figure 4. It is observed from this figure that for all of the joints, the maximum tracking error magnitudes are below 0.5 degrees which indicate the success of the sliding mode controller. 
The variations of the magnitudes of the interaction forces $F_{1}$ and $F_{2}$ versus time are given in Figure 5. Both forces have zero magnitudes during the $0 \leq t \leq 2$ time interval since there are no interaction between the load and the robot arms during the approaching motion. Then, in the second part of the motion, the load is handled and forces start to act. At the end of the motion it is observed that there exists residual force both for $F_{1}$ and $F_{2}$ which is due to the weight of the load. In fact the sum of these residual forces is equal to $m g / \mu$.

The joint torques, which are produced by sliding mode controller and acting on the related joints, are presented in Figure 6 . The changes in the torque magnitudes at $t=2 \mathrm{~s}$ is due to the load handling which produces interaction forces at the contact point between the load and the tip of the robot arms. The non-chattering control torque action is realized during the load approaching and transportation action as a result of proposed sliding mode control method.

\section{CONCLUSION}

In this study, a dual arm robot system for load transportation in hazardous environments was investigated. First, physical model of the robot was presented. The system consists of two planar arms cooperating in a horizontal plane. The friction forces arise on the contact points of the robot arm tips and load surface. Secondly, the non-chattering sliding mode control method was presented, which is used to produce the joint torques. Since, reliability and robustness are key features that a controller is supposed to posses, this control method was preferred in this study. Then, numerical results were presented. These results have shown that the sliding mode controller performed perfectly since, the robot arms tracked their given trajectory successfully while handling and transporting the load to its new location.

\section{APPENDIX}

i) Numerical parameters of the two-arm robot system

$$
\begin{array}{llll}
m_{i}=1.1 & {[\mathrm{~kg}]} & k_{i}=0.55 & {[\mathrm{~m}]} \\
I_{i}=0.1109 & {\left[\mathrm{kgm}^{2}\right]} & m=1.2 & {[\mathrm{~kg}]} \\
L_{i}=1.1 & {[\mathrm{~m}]} & b_{i}=110 & {[\mathrm{Nms}]} \\
\mu & =0.3 & &
\end{array}
$$

ii) Constant coefficients $A_{i}$ used in the equations of motion of the robot arms:

$$
\begin{aligned}
& A_{1}=m_{1} k_{1}^{2}+m_{2} L_{1}^{2}+I_{1} \\
& A_{2}=m_{2} k_{2}^{2}+I_{2} \\
& A_{3}=m_{2} L_{1} k_{2} \\
& A_{4}=m_{3} k_{3}^{2}+m_{4} L_{3}^{2}+I_{3}
\end{aligned}
$$




$$
\begin{aligned}
& A_{5}=m_{4} k_{4}{ }^{2}+I_{4} \\
& A_{6}=m_{4} L_{3} k_{4}
\end{aligned}
$$

iii) Numerical parameters of the controller:

$$
\tau_{i}=0.001 \quad \Gamma_{i}=500 \quad \lambda_{i}=1
$$

iv) The initial and final coordinates of the load:

$$
\begin{aligned}
& \left(x_{i}, y_{i}\right)=(0.1 .6) \quad[\mathrm{m}] \\
& \left(x_{f}, y_{f}\right)=(0.4,1.7)[\mathrm{m}]
\end{aligned}
$$

\section{REFERENCES}

[1] Zheng, Y.F. and Luh, J.Y.S., "Optimal load distribution for two industrial robots handling a single object", IEEE International Conference on Robotics and Automation, Philadelphia, USA, 344-349, (1988).

[2] Kron, A. and Schmidt, G., "Haptic telepresent control technology applied to disposal of explosive ordnances: Principles and experimental results", IEEE International Symposium on Industrial Electronics, Dubrovnik, Croatia, 15051510, (2005).

[3] Carignan, C.R. and Akin, D.L., "Cooperative control of two arms in the transport of an inertial load in zero gravity", IEEE Transactions on Robotics and Automation, 4(4), 414-419, (1988).

[4] Uchiyama, M., Iwasawa, N. and Hakomori, K., "Hybrid position/Force control for coordination of a two-arm robot", IEEE International Conference on Robotics and Automation, Raleigh, USA, 1242-1247, (1987).

[5] Laroussi, K., Hemami, H. and Goddard, R.E., "Coordination of two planar robots in lifting", IEEE Journal of Robotics and Automation, 4(1), 77-85, (1988).

[6] Utkin, V.I., "Variable structure systems with sliding modes", IEEE Transactions on Automatic Control 22, 212-222, (1977).

[7] Yagiz, N. and Hacioglu, Y., "Fuzzy Sliding Modes with Moving Surface for Robust Control of a Planar Robot", Journal of Vibration and Control, 11(3), 903-922, (2005).

[8] Corradini, M.L. and Orlando, G., "Control of mobile robots with uncertainties in dynamical model: a discrete time sliding mode approach with experimental results", Control Engineering Practice, 10, 23-34, (2002). 
[9] Herrmann, G., Spurgeon, S.K. and Edwards, C., "A model-based sliding mode control methodology applied to the HDA-plant", Journal of Process Control, 13, 129-138, (2003).

[10] Jafarov, E.M. and Tasaltin, R., "Robust sliding mode control for the uncertain MIMO aircraft model F-18", IEEE Transactions on Aerospace and Electronic Systems, 36(4), 1127-1141, (2000).

[11] Ertugrul, M., Kaynak, O. and Sabanovic, A., "A comparison of various vss techniques on the control of automated guided vehicles". In Proceedings of the IEEE International Symposium on Industrial Electronics, July 10-14, Athens, Greece, pp. 837-842, (1995).

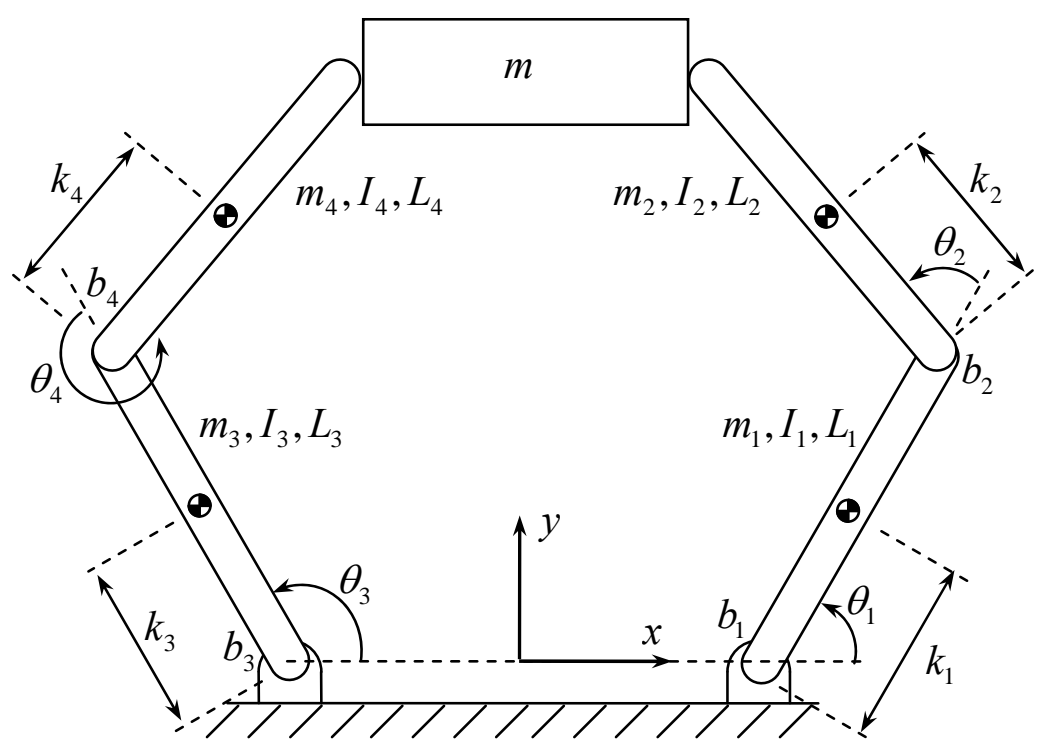

Figure 1 Physical model of the robot arms 


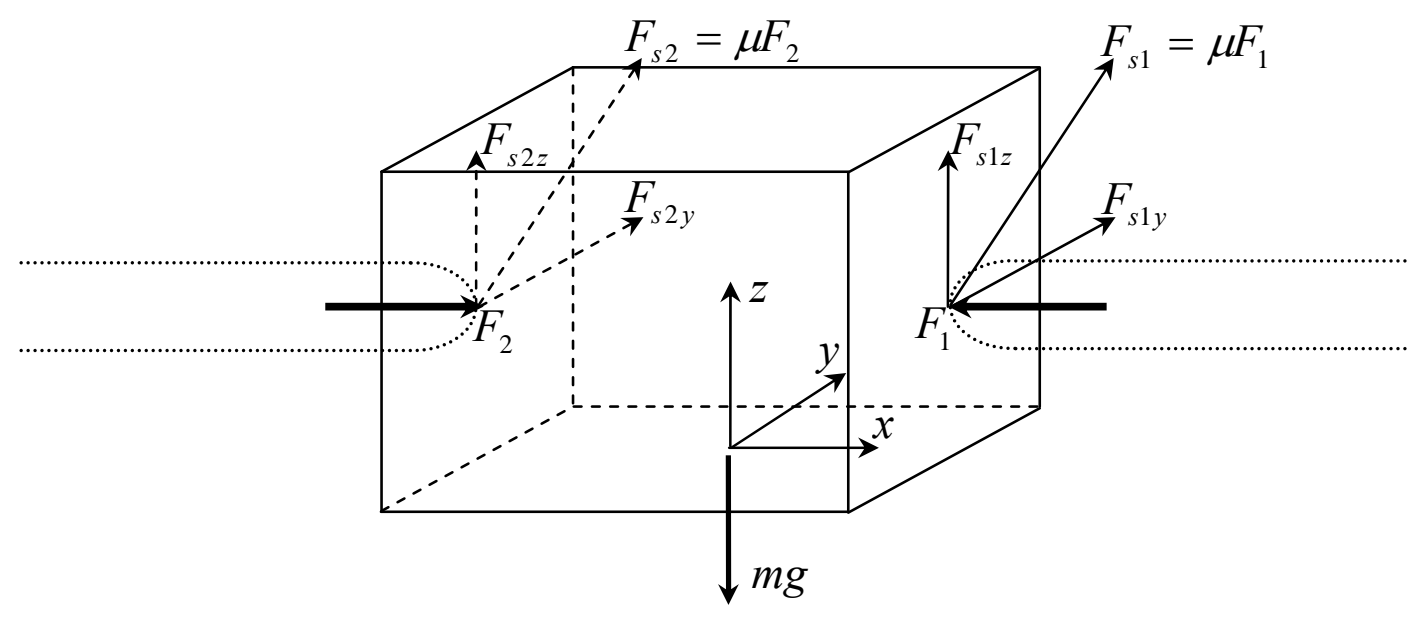

Figure 2 Representation of the forces acting on the load
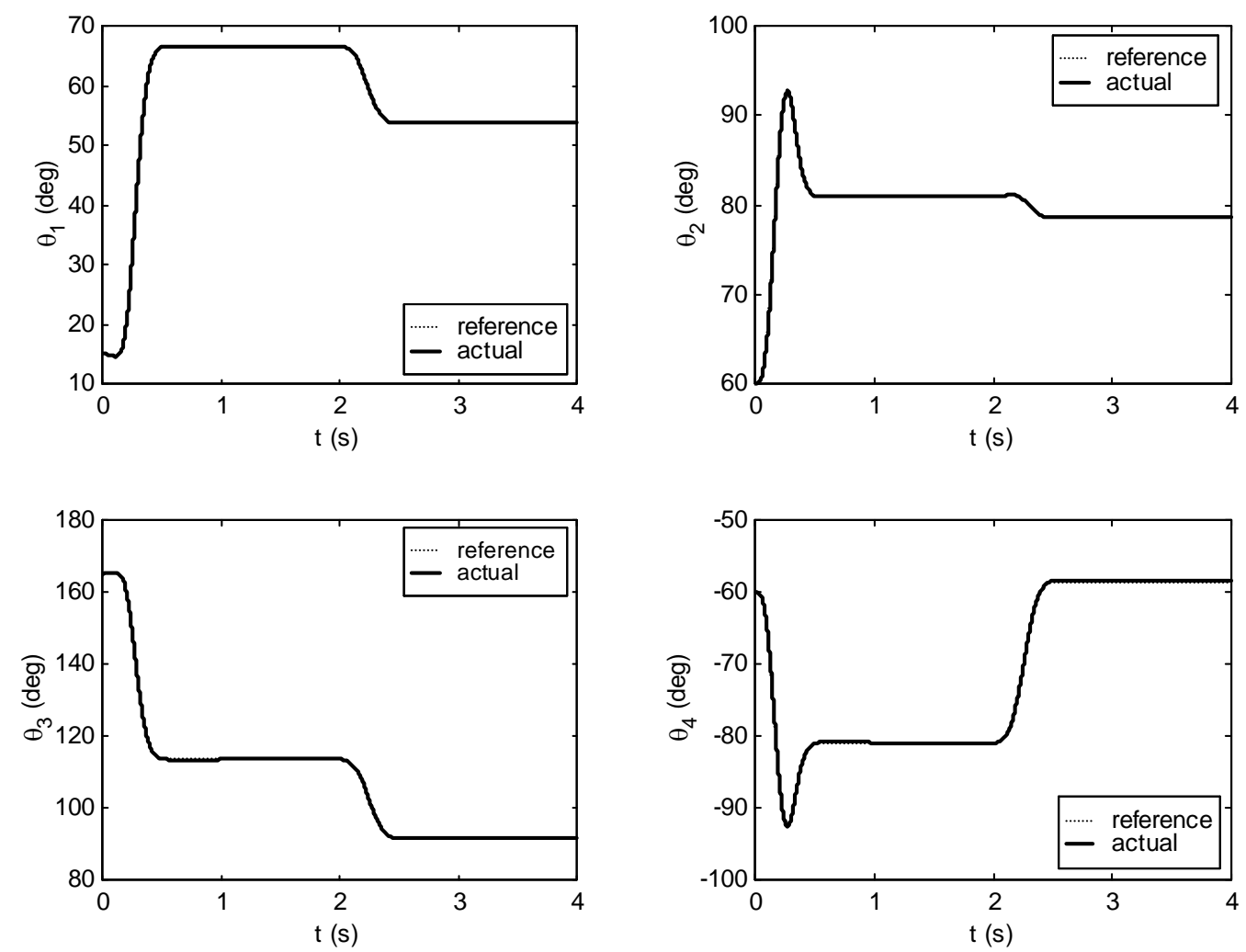

Figure 3 The reference and the actual joint angles 

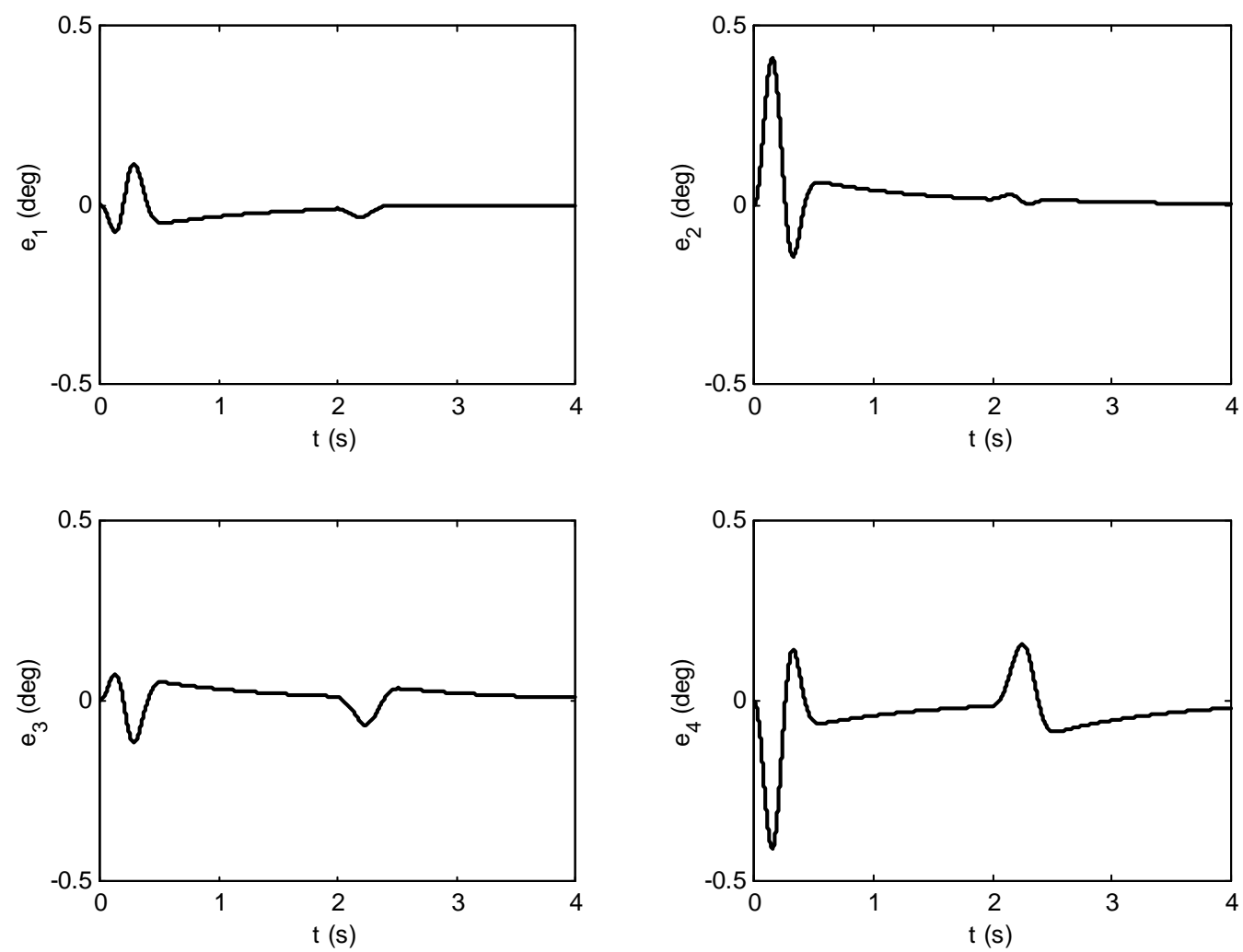

Figure 4 Tracking errors of the related joint angles
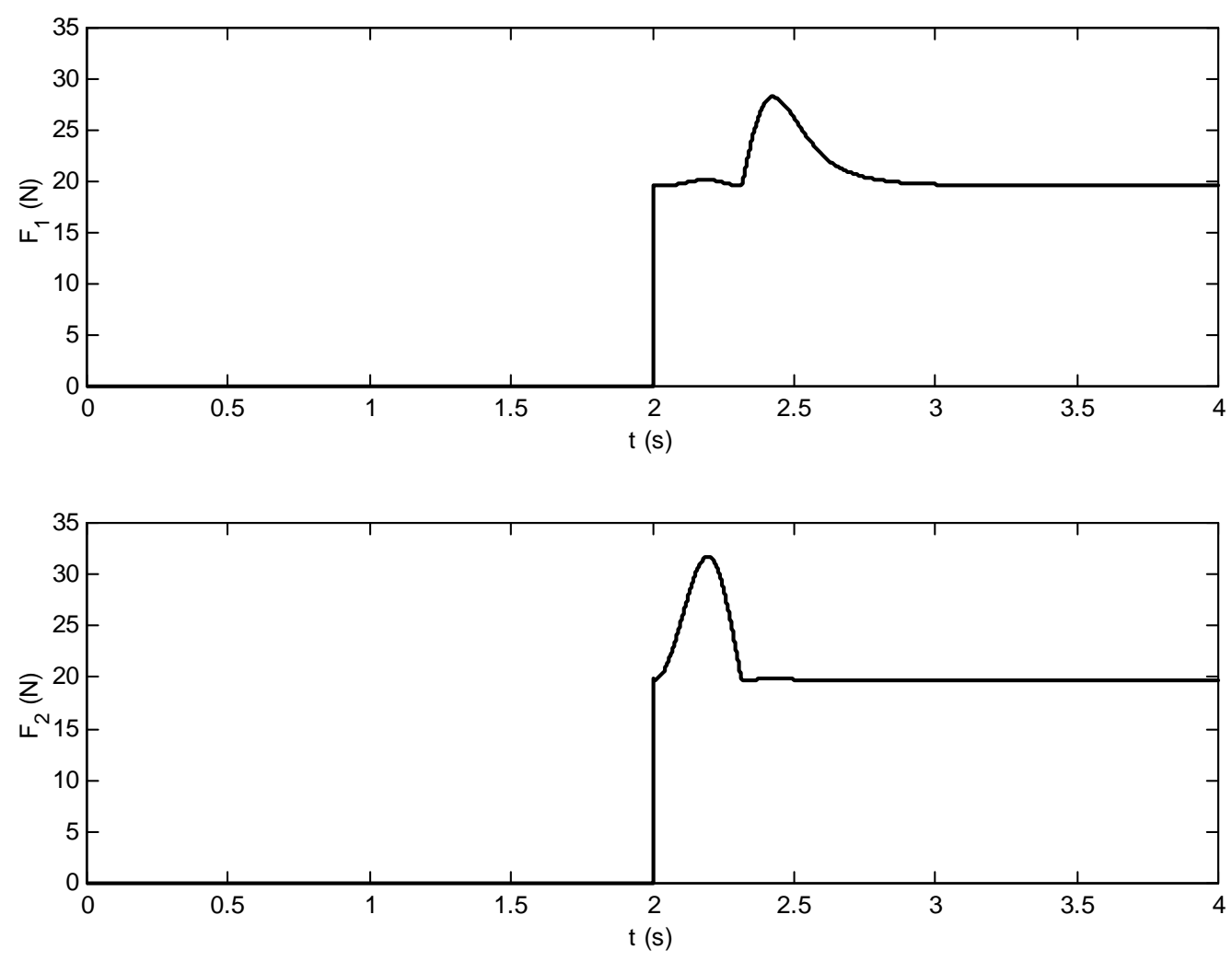

Figure 5 Interaction forces 

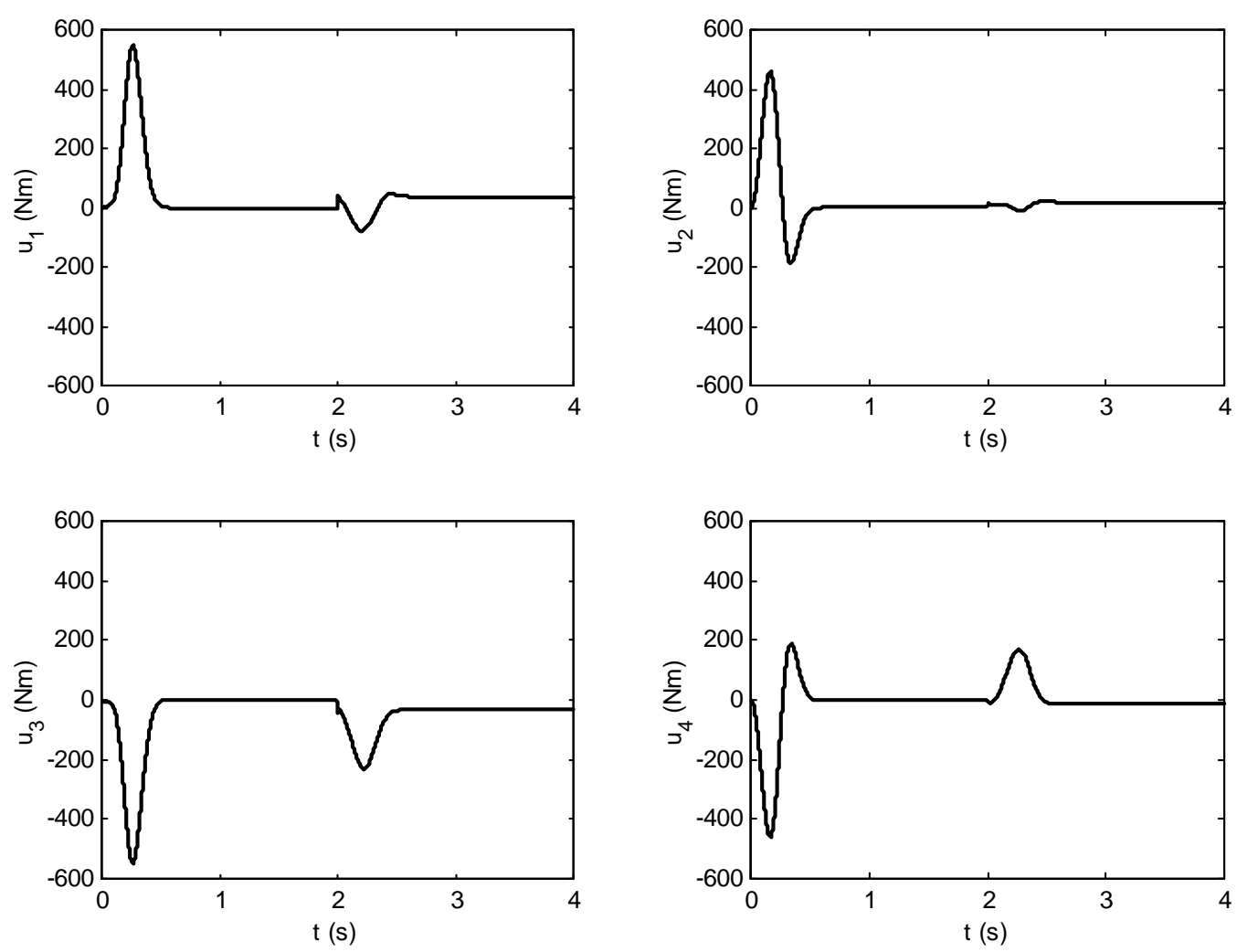

Figure 6 Applied joint torques 